Beobachtungen des Kometen $1911 \mathrm{~g}$ (Beljawsky)

mit dem $360 \mathrm{~mm}$ Refraktor der Universitäts-Sternwarte Kopenhagen angestellt von E. Strömgren.

\begin{tabular}{|c|c|c|c|c|c|c|c|c|c|c|}
\hline I 9 I I & M.Z.Kop. & $\Delta \alpha$ & $A \delta$ & Vgl. & $\alpha$ app. & $\log p \cdot A$ & $\delta$ app. & $\log p \cdot A$ & Red. ad I. app. & * \\
\hline & $17^{\mathrm{h}} 12^{\mathrm{m}} 40^{\mathrm{s}}$ & $+16^{m_{1}}$ & $3^{\prime}$ & & $10^{\mathrm{h}} 5$ & & $+8^{\circ} .5$ & & . & \\
\hline 3 & $\begin{array}{lll}17 & 18 & 37\end{array}$ & +822.41 & -0 12.8 & 5,5 & $\begin{array}{lll}\text { I I } & 37 & 56.30\end{array}$ & 9 . & + I I 3042 & & $65-3.5$ & 2 \\
\hline 4 & $17 \quad 1754$ & + 1029.89 & +25 & 3,3 & I I $50 \quad 45.67$ & & +11555 & & $64-3.9$ & \\
\hline 6 & 910 & $-55^{2.10}$ & to 2.8 & 5,5 & $12 \quad 1753.35$ & $9 \cdot 53^{\circ} \mathrm{n}$ & +1219 & 0.852 & $+0.59-5.1$ & 4 \\
\hline
\end{tabular}

Sept. 29. Rektaszension ziemlich unsicher.

\begin{tabular}{|c|c|c|c|}
\hline & & & Mittlere Örter \\
\hline$*$ & $\alpha \operatorname{Ig} 11.0$ & $\delta 1911.0$ & Autorität \\
\hline I & $10^{h} 3^{6^{m}} 43^{s}: 87$ & $+9^{\circ} I^{\prime} 40^{\prime \prime} 4$ & AG Lpz II $555^{2}$ \\
\hline 2 & $\begin{array}{lll}11 & 29 & 33.24\end{array}$ & +I I 3059.1 & AG Lpz I $4.33^{\circ}$ \\
\hline
\end{tabular}

Kopenhagen, Universitäts-Sternwarte, I 9 I I Okt. 26.

\begin{tabular}{|c|c|c|c|}
\hline$*$ & $\alpha 1911.0$ & $\delta 1911.0$ & Autorität \\
\hline 3 & I I ${ }^{\mathrm{h}} 40^{\mathrm{m}} 15^{5} \mathrm{I} 4$ & $+11^{\circ} 53^{\prime} 5^{\prime \prime} 7$ & AG Lpz I $43^{84}$ \\
\hline 4 & I $2 \cdot 23 \quad 44.86$ & +12196.4 & $45^{88}$ \\
\hline
\end{tabular}

Elis Strömgren.

\title{
Zum Problem der approximativen Berechnung allgemeiner Störungen der kleinen Planeten. Vergleich zwischen Beobachtung und Rechnung für 24 Themis. Von Elis Strömgren.
}

Die Zahl der Neuentdeckungen von kleinen Planeten ist bekanntlich in den letzten Jahren so gewaltig angewachsen, $\mathrm{daß}$ die genaue Verfolgung aller dieser Himmelskörper zu einer Unmöglichkeit geworden ist.

Von umfassenden Berechnungen allgemeiner Störungen liegen in den letzten Jahren nur ganz vereinzelte Arbeiten vor. Hieran läßt sich wahrscheinlich vorläufig nichts ändern, da in der Astronomie in unserer Zeit ganz andere Richtungen bevorzugt werden; es ist aber zu hoffen, daß einmal in der Zukunft ein Teil der disponiblen Arbeitskräfte wieder zu diesem schönen aber mühevollen Arbeitsgebiete der Astronomie zuriickkehren werde.

Wenn die Aussichten auf dem Gebiete der exakten Berechnung allgemeiner Störungen also augenblicklich nicht die besten sind, ebenso wie an eine systematische Verfolgung der kleinen Planeten durch Berechnung spezieller Störungen nicht mehr zu denken ist, so ist andererseits doch zu hoffen, daß eine nicht allzu ferne Zukunft uns wichtige Resultate auf dem Gebiete der approximativen Berechnung allgemeiner Störungen bringen wird. Für die theoretische Behandlung dieses Problems ist schon eine beträchtliche Arbeit geleistet worden, vor allen Dingen von Backlund, Bohlin und Brendel. In bezug auf die Verwertung dieser Untersuchungen für numerische Rechnungen sind besonders die Pulkowoer und die Stockholmer Arbeiten zu erwähnen. Ich beschränke mich hier darauf, einige Worte über die letzteren zu sagen. Bohlin selbst hat seine Theorie für einige Planeten des Typus I : 3 verwertet; für den Typus $\mathbf{1}: 2$ hat $v$. Zeipel eine mühsame Berechnung von Tafeln ausgeführt und letztere mit Erfolg auf einige konkrete Fälle angewandt, und für die Saturnstörungen einer größeren Planetengruppe hat Block Tafeln berechnet. Es kann wohl kaum bezweifelt werden, daß diese Arbeiten in der Zukunft großen Nutzen bringen werden.

1) Astron. Nachrichten 4123.
Unter der Leitung von Leuschner hat die amerikanische National Academy of Sciences kürzlich eine umfassende Arbeit über die Watsonschen Planeten herausgegeben, die zu diesem Gebiete gehört.

Als ein Beispiel zur Bohlinschen Theorie hat der Unterzeichnete vor einigen Jahren ${ }^{1}$ ), unter Anwendung der von v. Zeipel für die Hekubagruppe gegebenen Formeln und Tafeln die Störungen des Planeten 24 Themis berechnet. Als Grundlage dienten die von $A$. Krueger ausgeführten Rechnungen über Themis. Diese erstrecken sich über einen Zeitraum von vierundeinhalb Jahrzehnten, bis 1898 ; sie waren nachher von $\mathcal{F}$. Moller und mir fortgesetzt worden und bis zur Opposition rgo8 unter genauer Berechnung der speziellen Störungen weitergeführt. Der in dem zitierten Aufsatze (A. N. 4123) gegebene Vergleich zwischen meinen Tafeln und dem für I 905 Juni 27.0 M. Z. Berlin oskulierenden Elementensysteme muß als eine Bestätigung der praktischen Verwendbarkeit der Bohlinschen Theorie betrachtet werden. Seitdem sind sechs Jahre vergangen, und ich habe gemeint, daß der fortgesetzte Vergleich zwischen Beobachtung und Rechnung eine nützliche Arbeit sei. Dieser Vergleich wird für die Zeit bis zur Opposition I9I I inkl. im vorliegenden Artikel gegeben.

$\mathrm{Zu}$ den A. N. 4 I 23 gegebenen Formeln und Tafeln ist folgendes zu bemerken. Die Koeffizienten sind in Bogensekunden gegeben. Da es für die Aufgaben, von denen hier die Rede ist, zweckmäßiger erscheint, alles in Bogenminuten ausgedrückt zu haben, gebe ich in der folgenden Zusammenstellung alle Koeffizienten in Bogenminuten an. Ferner ist zu bemerken, daß die Koeffizienten $k_{2}$ in der Tafel für die dritte Koordinate (A. N. 4 II3 p. 298) irrtümlich als für $u$ gültig angegeben sind. Die in der Tafel gegebenen Zahlen geben in der Tat die Koeffizienten der Entwickelung von $(u / c)$ sec $i$ statt $u$ (die Koeffizienten $k_{2}^{\prime}$ sind dagegen richtig). 
Es handelt sich aber nur un einen Schreibfehler im Manuskript; die Rechnungen, die mit den Zahlenwerten dieser Tafeln ausgeführt sind, sind alle richtig.

In der folgenden Zusammenstellung ist dieser Fehler korrigiert worden. Wir erhalten, in derselben Bezeichnung wie der A. N. 4123 angewandten:

Mittlere Elemente.

Epoche 1874 Dez. 6.0 M. Z. Berlin.

$$
\begin{aligned}
& n=639^{\prime \prime} 5722 \\
& \varphi=7^{\circ} 25^{\prime} 55 \\
& c=-10^{\circ} 17^{\prime} 12
\end{aligned}
$$

\begin{tabular}{|c|c|c|c|c|c|c|c|}
\hline \multicolumn{2}{|c|}{$\chi$} & \multicolumn{2}{|c|}{$n d z-[n \delta z]$} & \multicolumn{2}{|r|}{$\nu$} & \multicolumn{2}{|c|}{$u$} \\
\hline$i$ & $j$ & $\log k$ & $K$ & $\log k_{1}$ & $K_{1}$ & $\log k_{2}$ & $K_{2}$ \\
\hline 0 & $\circ$ & - & - & 9.9484 & $0^{\circ}$ o.'o & 7.026 & $270^{\circ} \quad 0^{\prime} 0$ \\
\hline $\mathbf{I}$ & I & 0.9143 & $308^{\circ} 56.8$ & 0.3679 & $127 \quad 38.3$ & $7 \cdot 742$ & 97 I 3 \\
\hline 2 & 2 & 1.7500 & $25^{6} \quad 13-3$ & 1.47724 & $76 \cdot 18.0$ & 8.839 & $306 \quad 35$ \\
\hline 3 & 3 & $0.309 \mathrm{I}$ & 2 I $\quad 3$ I. 4 & 0.2003 & $202 \quad 53.6$ & - & - \\
\hline 4 & 4 & 9.9 I 52 & I 6 I 14.7 & $9.4 \times 6$ & $3033^{2}$ & - & - \\
\hline 5 & 5 & 9.2544 & $278 \quad 9.6$ & 9.167 & $98 \times 0$ & - & - \\
\hline 3 & I & - & - & 9.234 & $1273^{8}$ & 8.408 & 31059 \\
\hline 4 & 2 & 0.2818 & $75 \quad 15.9$ & - & - & - & \\
\hline-2 & 0 & 9.8222 & I $88 \quad 25.6$ & 8.680 & 1800 & 8.103 & 17639 \\
\hline$-\mathbf{I}$ & I & $0.265^{6}$ & $324 \quad 19.5$ & 9.3734 & $\begin{array}{ll}339 & 23.5\end{array}$ & 8.702 & $304 \quad 17$ \\
\hline 0 & 2 & - & - & I. 1204 & 79 I 5.8 & 8.705 & 7155 \\
\hline I & 3 & 1.1015 & $41 \quad 5.0$ & $0.65^{6} 3$ & $220 \quad 8.6$ & $8.75^{\mathrm{I}}$ & I $99 \quad 33$ \\
\hline 2 & 4 & 1.0820 & $348 \quad 19.4$ & 0.8070 & I 680.0 & 8.927 & I 47 I I \\
\hline 3 & 5 & 0.0766 & I 1650.0 & 9.9277 & $297 \quad$ I 5.7 & 7.701 & $274 \quad 49$ \\
\hline 4 & 6 & 9.3999 & $244 \quad 39.6$ & 9.2716 & $65 \quad 40.5$ & - & - \\
\hline 5 & 7 & $9.1 \times 9$ & $124 x$ & 9.5224 & I $79 \quad 30.8$ & -- & - \\
\hline-2 & 2 & 0.3001 & $291 \quad 23.3$ & 9.7113 & $33^{2} \quad 3.9$ & 9.4064 & $262 \quad 49.5$ \\
\hline$-r$ & 3 & 9.9217 & $230 \quad 49.9$ & 9.8230 & $225 \square 2.2$ & $8.59 \mathrm{I}$ & $335^{\circ}$ \\
\hline o & 4 & - & - & 0.637 I & $181 \quad 5^{2.5}$ & 8. I 44 & I $825 \mathrm{I}$ \\
\hline $\mathbf{I}$ & 5 & 0.71 .32 & I 3445.6 & 0.2278 & 3 I 444.2 & 8.540 & $295 \quad 8$ \\
\hline 2 & 6 & $0.6 \times 84$ & 8 I 18.6 & 0.3357 & 26138.9 & 8.519 & 2437 \\
\hline 3 & 7 & 9.3162 & 215 & 8.254 & I2I 20 & - & - \\
\hline o & 6 & - & - & $0.025^{2}$ & $277 \quad 9.8$ & - & -- \\
\hline 2 & 8 & 9.8830 & I 7948.0 & 9.8233 & $357 \quad 45.7$ & - & - \\
\hline & & $\log k^{\prime}$ & $K^{\prime}$ & $\log k_{1}{ }^{\prime}$ & $K_{1}^{\prime}$ & $\log k_{2}{ }^{\prime}$ & $K_{2}^{\prime}$ \\
\hline 2 & 0 & 1.2414 & $170^{\circ} 5^{2 !} \cdot 3$ & 0.9196 & $170^{\circ} 22,6$ & $8.498 \mathrm{I}$ & I $85^{\circ} 53^{\prime} .8$ \\
\hline$\circ$ & $\circ$ & - & - & 9.310 & 12738 & 7.6072 & $0 \quad 0.0$ \\
\hline 4 & o & 9.69 I I & $35^{\circ} \quad 5^{6.4}$ & - & - & -- & - \\
\hline$\circ$ & 2 & - & - & 9.186 & $265 \quad 27$ & - & - \\
\hline 2 & 4 & $9.123^{8}$ & $340 \quad 40.8$ & 8.826 & $340 \quad 48$ & 一 & - \\
\hline 4 & 2 & 8.8963 & $245 \quad 29.7$ & $8.73^{6}$ & $245 \quad 33$ & 一 & - \\
\hline
\end{tabular}

$$
\begin{aligned}
& \pi=\text { I } 39^{\circ} 43^{\prime} \cdot 47 \\
& \delta=3544.62 \text { 1 } 860.0 \\
& i=048.4 \mathrm{I}
\end{aligned}
$$

$$
\begin{aligned}
n d z-[n d z] & =\Sigma k \sin (\chi+K)+\left(\vartheta+189^{\circ} 44^{\prime}{ }^{\prime} 5\right) \cdot \Sigma k^{\prime} \cos \left(\chi+K^{\prime}\right) \\
\nu & =\Sigma k_{1} \cos \left(\chi+K_{1}\right)+\left(\vartheta+189^{\circ} 44^{\prime}{ }_{5}\right) \cdot \Sigma k_{1}^{\prime} \sin \left(\chi+K_{1}^{\prime}\right) \\
u & \left.=\Sigma k_{2} \sin \left(\chi+K_{2}\right)+T \cdot \Sigma k_{2}^{\prime} \cos \left(\chi+K_{2}^{\prime}\right)^{3}\right)
\end{aligned}
$$

wo $\chi=i 1 / 2 \varepsilon+j y$.

Die Koeffizienten dieser Tafel sind alle in Bogenminuten ausgedrückt.

1) Koeffizienten logarithmisch und in Bogenminuten.

?) Koeffizienten logarithmisch und linear.

:) $T=$ Julianische Jahre seit 1874 Dez. 6.o.

$$
\begin{aligned}
& {[n d z]_{1}=[2.360893] \sin \left(2 \zeta+261^{\circ} 42.88\right)} \\
& +[1.54742] \sin \left(4 \zeta+8^{\circ} 7^{\prime} \cdot 25\right) \\
& +[0.6829] \sin \left(6 \zeta+106^{\circ} 37^{\prime}\right) \\
& \left.+\left(\varepsilon+10^{\circ} \text { I }^{\prime} \text { I } 2\right)^{2}\right)\left[\left[0.088_{5} \mathrm{I}\right] \sin \left(2 \zeta-5^{\circ} 20^{\prime} 5\right)\right. \\
& \left.+\left(\varepsilon+10^{\circ} \text { I }^{\prime} !_{2}\right)^{2} \cdot[5.7 \text { 1 } 55]^{1}\right) \\
& \left.+[8.3728] \sin \left(4 \zeta+89^{\circ} 23^{\prime} 8\right)\right] \\
& \left.[n \delta z]=[n \delta z]_{1}+[6.817392 \mathrm{n}] \cdot \varepsilon+[8.3090] \cdot\left[n^{\prime} \delta z^{\prime}\right]^{2}\right) \\
& \left.\zeta=[8.504275] \cdot \varepsilon-189^{\circ} 24^{\prime} 2 \mathrm{I}-\left[9.995^{8}\right] \cdot\left[n^{\prime} \delta z^{\prime}\right]^{2}\right) \\
& \left.\left[n^{\prime} \delta z^{\prime}\right]=20.05 \sin \left(0^{\circ} .385 T+124^{\circ} \cdot 3\right)^{3}\right) \\
& \left.y=\zeta+[9.67002 \mathrm{I}] \cdot[n d z]_{1}\right)
\end{aligned}
$$


In den Oppositionen I906, I907-08, I909 und I9 I I liegen eine Anzahl guter Beobachtungen vor. Ich habe die folgenden zum Vergleich mit den oben gegebenen Formeln und Tafeln ausgewählt:

\begin{tabular}{|c|c|c|c|c|c|c|c|}
\hline $\mathrm{Be}$ & $\mathrm{n}-$ Aberr. & $\alpha$ app. & . & $\delta$ app. & & Geoz. $\alpha$ app. & Geoz. $\delta$ app. \\
\hline & 06 & $I^{h}$ & & $+8^{\circ} 32^{\prime}$ & & $\mathrm{I}^{\mathrm{h}} 2$ & $+8^{\circ}$ \\
\hline & I907 Dez. 3 & $734 \quad 4$ & - & $+225 I$ & & 734 & +22518.0 \\
\hline & 1908 Jan. 20. & 7 I 655 & 07 & +2329 1 3.9 & & 716 & +232916.6 \\
\hline & I909 Mai & 14457. & -0 & 一 I $243 \quad 54.2$ & +4.0 & I $4 \quad 4 \quad 57$ & $-12.43 \quad 5^{0.2}$ \\
\hline Kopent & I 9 I I Sept. & $2322 \quad 22.53$ & +0.04 & $-45^{8} 23.5$ & $+3 \cdot 1$ & $\begin{array}{lll}23 & 22 & 22.57\end{array}$ & $-45^{8} \quad 20.4$ \\
\hline
\end{tabular}

Die für die Berechnung der Parallaxe und der Aberrationszeit nötigen Werte der geozentrischen Distanz sind den exakten Ephemeriden bezw. den Oppositionsdaten im Berl. Jahrbuch entnommen.

Die Hauptdaten der mit den oben gegebenen Störungsausdrücken ausgeführten Berechnung der Örter des Planeten für die Beobachtungsepochen lauten:

\begin{tabular}{|c|c|c|c|c|c|}
\hline & r 906 Okt. I 4 & 1907 Dez. $3^{1}$ & I908 Jan. 20 & I 909 Mai 7 & I 9 I I Sept. 3 \\
\hline $\begin{array}{c}\varepsilon \\
\zeta \\
y \\
{[n \delta z]_{1}} \\
{[n \delta z]-[n \delta z]_{1}}\end{array}$ & $\begin{array}{c}10 \pi+248^{\circ} 22 ! 308 \\
-124^{\circ} 12.85 \\
-12357.10 \\
+33^{\prime} 679 \\
-\quad-80.436\end{array}$ & $\begin{array}{c}\text { I0 }+330^{\circ} 41^{\prime} .426 \\
-121^{\circ} 34.77 \\
-121 \text { I I.I } 2 \\
+50.553 \\
-83.686\end{array}$ & $\begin{array}{c}10 \pi+334^{\circ} 46.095 \\
-\mathrm{I} 2 \mathrm{I}^{\circ} 26^{\prime} 97 \\
-\mathrm{I} 2 \mathrm{I} 2.93 \\
+5 \mathrm{I} \cdot 385 \\
-83.847\end{array}$ & $\begin{array}{c}12 \pi+70^{\circ} \mathrm{I} 9^{\prime} \cdot 420 \\
-\mathrm{I} \times 8^{\circ} 24^{\prime} .02 \\
-\mathrm{I} I 750.82 \\
+70.97 \mathrm{I} \\
-87.609\end{array}$ & $\begin{array}{c}\text { I } 2 \pi+209^{\circ} 54^{\prime} 593 \\
- \text { I } 13^{\circ} 56^{\prime} .2 \mathrm{I} \\
- \text { I } 139.64 \\
+99^{\prime} 55^{8} \\
\text { - } 93.1 \mathrm{I} 7\end{array}$ \\
\hline 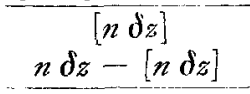 & $\begin{array}{r}-46.757 \\
-38.518 \\
\end{array}$ & $\begin{array}{r}33^{\prime} \mathrm{I} 33 \\
-30.522 \\
\end{array}$ & $\begin{array}{r}32.462 \\
-27.815 \\
\end{array}$ & $\begin{array}{r}16.638 \\
+\quad 39.880 \\
\end{array}$ & $\begin{array}{r}+6.441 \\
-\quad 19.523 \\
\end{array}$ \\
\hline $\begin{array}{c}n \delta z \\
\nu \\
u\end{array}$ & $\begin{array}{r}1^{\circ} 25 \cdot 275 \\
+0.0016706 \\
+0.519\end{array}$ & $\begin{array}{c}-I^{\circ} 3.655 \\
-0.0084226 \\
-0.576\end{array}$ & $\begin{array}{c}1^{\circ} 0^{\prime} 277 \\
-0.0087760 \\
-0.608\end{array}$ & $\begin{array}{c}+23.242 \\
-0.0058542 \\
-0.184\end{array}$ & $\begin{array}{r}13.082 \\
+0.0030980 \\
+0.915\end{array}$ \\
\hline $\log r$ & $0.5170 \mathrm{I}$ & 0.44047 & 0.43825 & 0.47420 & 0.54354 \\
\hline $\begin{array}{l}l_{1860.0} \\
b_{1860.0}\end{array}$ & $\begin{array}{r}2 \mathrm{I}^{\circ} 2 \mathrm{I} .24 \\
-0 \mathrm{II} .54 \\
\end{array}$ & $\begin{array}{r}106^{\circ} 33^{\prime} \cdot 37 \\
+0 \quad 45 \cdot 07 \\
\end{array}$ & $\begin{array}{r}111^{\circ} 750 \\
+046.16 \\
\end{array}$ & $\begin{array}{rr}217^{\circ} & 11.80 \\
-0 & 1.42 \\
\end{array}$ & $\begin{array}{r}346^{\circ} 7^{\prime} .66 \\
-0 \quad 3^{6.05} \\
\end{array}$ \\
\hline $\begin{array}{l}b_{1910.0} \\
b_{1910.0} \\
\end{array}$ & $\begin{array}{rr}22^{\circ} 3^{\prime} \mathrm{I} 2 \\
-0 \quad 11.65 \\
\end{array}$ & $\begin{array}{r}107^{\circ} \mathrm{I} 5^{\prime 25} \\
+044.68 \\
\end{array}$ & $\begin{array}{l}\text { I I I } 499^{\prime} 3 \\
\text { +o } 45.78 \\
\end{array}$ & $\begin{array}{rr}217^{\circ} & 53^{\prime} 68 \\
-0 & 1.22 \\
\end{array}$ & $\begin{array}{r}346^{\circ} 49^{\prime} .54 \\
-0 \quad 35.92 \\
\end{array}$ \\
\hline
\end{tabular}

Hieraus ergibt sich, nachdem wir die so berechneten $\alpha$ und $\delta$ auf das wahre Äquinoktium bezogen haben, folgendes Resultat:

\begin{tabular}{|c|c|c|c|c|c|c|}
\hline & \multicolumn{2}{|c|}{ Beob. } & \multicolumn{2}{|c|}{ Rechn. } & \multicolumn{2}{|c|}{$B-R$} \\
\hline & Geoz. $\alpha$ app. & Geoz. $\approx$ app. & $\alpha$ & $\delta$ & $\Delta \alpha \cos \delta$ & $\Delta \delta$ \\
\hline I906 Okt. I 4 & $20^{\circ} 58.62$ & $+8^{\circ} 32.05$ & $2 \mathrm{I}^{\circ} \quad 0^{\prime} 35$ & $+8^{\circ} 32 ! 25$ & - I' ${ }^{\prime} \mathbf{I}$ & - o' 20 \\
\hline 1907 Dez. 31 & I I 340.86 & +225 I.I 3 & I I 338.46 & +2250.04 & $+2.2 \mathrm{I}$ & +1.09 \\
\hline 1908 Jan. 20 & $109 \mathrm{I} 3.9 \mathrm{I}$ & +2329.28 & I09 I I.80 & +2328.07 & +1.94 & +1.21 \\
\hline I909 Mai & 2 I 194.46 & -1243.84 & $211 \quad 11.97$ & -1241.83 & +2.43 & $-2.0 \mathrm{I}$ \\
\hline I9I I Sept. 3 & $35^{\circ} \quad 35.64$ & -458.34 & $35^{\circ} \quad 3^{8.4} \mathrm{I}$ & -456.84 & -2.76 & $\therefore \mathrm{I} .50$ \\
\hline
\end{tabular}

Die Übereinstimmung zwischen Beobachtung und Rech- Störungen erster Ordnung in bezug auf die störende Masse nung muß als eine sehr gute bezeichnet werden, besonders wenn beachtet wird, daß das letzte für die Berechnung der Störungsausdrücke angewandte im Jahre I 897 oskulierende Elementensystem schon so weit zurückliegt, daß ferner nur berechnet sind, und daß wir die Saturnstörungen überhaupt nicht berücksichtigt haben.

Zum Aufsuchen des Planeten scheinen jedenfalls die gegebenen Störungsausdriicke für längere Zeiten zu genügen.

Kopenhagen, Universitäts-Sternwarte, I 9 I I Dezember.

Elis Stromgren.
1) A. N. 4165 .
2) A. N. 4233 .
3) A. N. 4295 .
4) A. N. 4396 .
5) Von Herrn Observator Pechüle mitgeteilt. 